Stefan Kubica, Hagen Ringshausen, Jörg Reiff-Stephan, Marius Schlingelhof (Hrsg.) 2. Automobil Symposium Wildau:

Tagungsband Technische Hochschule Wildau 2017

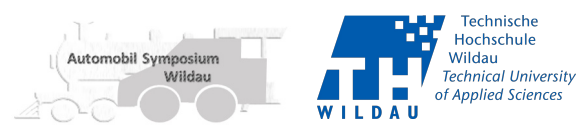

Günter Tolkiehn gt@tolkiehn-partner.de

Peter Weigert

peter.weigert@gmx.net

Christian Lührs

cbristian@mc-luebrs.de

\title{
ÖPNV 4.0
}

\section{Zusammenfassung}

Die globalen Technologietrends Elektroantrieb und fahrerloses Fahren ermöglichen in den nächsten Jahren das Eindringen des ÖPNV in den bisher von Privatfahrzeugen dominierten Individualverkehrssektor. Die Gestaltung dieser Transformation ist von weitreichender verkehrs- umwelt-, ordnungs- und industriepolitischer Bedeutung. Eines der hierfür zu diskutierenden Szenarien ist ÖPNV 4.0, die Ergänzung des ÖPNV um den gemanagten Betrieb von Schwärmen kleiner, fahrerloser EStraßenfahrzeuge.

ÖPNV 4.0 ermöglicht einen individuellen 24/7-end-to-end-service durch neue

- intermodale Angebote, die nach individuellem Bedarf die gesamte Wegstrecke einschließlich der ersten und letzten Meile abdecken

- monomodale Angebote, z.B. für Strecken und Zeiten weit außerhalb des Wirtschaftlichkeitsbereiches der Massenverkehrsmittel (schwach erschlossene Gebiete, Betriebsruhezeiten, Linienverkehre mit großer Taktung und geringer Auslastung)

Sofern es gelingt den politischen Willen aufzubringen, der Entwicklung des doi: 10.15771/ASW_2017_1
ÖPNV für die nächsten 15 Jahre diese Richtung zu geben, kann dies weitreichende positive Auswirkungen haben:

- Individuelle Mobilität wird nachhaltig und zu einem Bruchteil des bisherigen Aufwands für jedermann verfügbar („Volks-Wagen“)

- Das individualisierte öffentliche Mobilitätsangebot ruft einen erheblichen Rückgang der Haltung privater PKW hervor - mit allen wünschenswerten Folgen, wie Innovationsschübe von globaler Bedeutung für TelematikRobotik- und Fahrzeugindustrie, Reduktion von Umweltbelastung, Staus, Parkflächenbedarf, Unfallgefahr, Kapitalbindung.

- Der gesamte Transformationsprozess einschließlich der Nutzung der anfallenden Kundendaten bleibt auf Grund der überwiegend staatlichen Betreiber marktwirtschaftlich und ordnungspolitisch beherrschbar

Der Beitrag gibt einen Überblick über technische, wirtschaftliche, zeitliche und unternehmerische Eckdaten und Herausforderungen für ÖPNV 4.0. Alternative Entwicklungsszenarien werden gegenübergestellt. 


\section{Einleitung}

In den hochindustrialisierten Ländern sowie auch in vielen Schwellenländern finden wir heute leistungsfähige Verkehrssysteme. Sie setzen typischerweise auf passiven Infrastrukturen (Straßennetz, Schienennetz, Wasserwege, Landebahnen) auf. Hinzu kommen aktive Infrastrukturelemente wie Tankstellen, Verkehrsleitsysteme, Parkplätze, Bahnhöfe, Fahrstrom- und Signalnetze, Häfen, Flughäfen etc. Weitere Elemente sind natürlich die eigentlichen Fahrzeuge.

Der Personenverkehr in Deutschland wird heute klar vom sog. Motorisierten Individualverkehr (MIV) dominiert. Sein Anteil an der gesamten jährlichen Beförderungsleistung von etwa $1200 \mathrm{Mrd}$. verkehr des ÖPNV 3.0 nutzen dabei gemeinsam die Straßeninfrastruktur, die damit heute Basis von etwa $85 \%$ der Beförderungsleistung und von über $90 \%$ der zurückgelegten Wegstrecken ist [Hütt13]. Die sog. Massenverkehrsmittel spielen dagegen, vor allem im Nahverkehr, heute nur noch eine nachgeordnete Rolle. Der von den heutigen Zahlenverhältnissen auf den Kopf gestellte begriffliche Unterschied hat in der Nutzerperspektive ohnehin keine Begründung. Der Mobilitätsbedarf ist unabhängig von der genutzten Technik ganz überwiegend individuell.

Aus der langen Tradition heraus haben sich aber zu diesem Begriffspaar neben der Korrelation „Massenverkehr - öf fentlich“, weitere Unterschiede bzw. Zuschreibungen eingebürgert (Tabelle 1).

\begin{tabular}{|l|l|l|}
\hline & „Individualverkehr“ & „Massenverkehr“" \\
\hline Fahrzeugeigentum & Nutzer & Verkehrsunternehmen \\
\hline Infrastruktureigentum & Staat, Privatunternehmen & Verkehrsunternehmen/Staat \\
\hline Zeiten & Freie Fahrzeitwahl 24/7 & Fahrplangebundenes Angebot \\
\hline Wege & $\begin{array}{l}\text { Typischerweise monomodal } \\
\text { (aber: Parkplatzproblem) }\end{array}$ & $\begin{array}{l}\text { Haltestellen und Fahrstrecken } \\
\text { vorgegeben, multimodales Netz }\end{array}$ \\
\hline Fahrweg & Freie Routenwahl & Routenvorgabe \\
\hline Zuverlässigkeit & Hoch (Einschränkung: Staus) & Hoch \\
\hline Kosten & $\begin{array}{l}\text { Mischkalkulation aus Treibstoff- } \\
\text { verbrauch und festen Kosten }\end{array}$ & $\begin{array}{l}\text { Granular nach Verbrauch } \\
\text { (Einzeltickets) oder Flatrate }\end{array}$ \\
\hline
\end{tabular}

Tabelle 1: Unterschiede Mobilitätsbedarf

Personenkilometern betrug 2010 bereits über 75\% [Hütt13]. Der MIV übertrifft damit deutlich die Verkehrs-leistungen aller sonstigen Verkehrsarten zusammengenommen, insbesondere auch die der früher zu Recht so genannten Massenverkehrsmittel [Beck11] des heutigen ÖPNV 3.0. Seit 1950 hat sich die Transportleistung des MIV auf das dreißigfache erhöht. Daran haben die mittleren Wegstrecken den wesentlichen Anteil, während sich die Anzahl der Wege je Person und Tag und auch die Wegezeiten nicht wesentlich erhöht haben. MIV, Fußgänger, Radfahrer und der Bus-
Dieser historische Hintergrund und die damit verbundenen Denkschemata erschweren heute gelegentlich den unbefangenen Blick auf disruptive Entwicklungsoptionen und innovative Mobilitäts-Szenarien. Heute sehen wir im PKW-Bereich drei grundlegende, technologiebasierte und globale Entwicklungstrends, die gleichzeitig auch das Potenzial haben, wesentlich zur Lösung von mehreren schwerwiegenden Entwicklungsproblemen im Verkehrswesen beizutragen. Diese drei Entwicklungstrends sind: 
1 Weg vom fossilen Brennstoff - Hin zu erneuerbaren Energiequellen

2 Weg vom menschlichen Fahrer - Hin zum fahrerlosen Fahrzeug

3 Weg vom privaten PKW - Hin zur on-demand Nutzung als Mobility-asa-Service (MaaS)

Diese drei Trends laufen zeitlich fast parallel, aber nicht rein technologiegetrieben und auch nicht völlig unabhängig voneinander. Tatsächlich spielen dabei auch Probleme des zunehmenden MIV eine Rolle, für die man sich durch Innovationen Lösungen erhofft, wie:

- Umweltbelastung durch Emissionen (CO2, NO2 [BrPe17], Feinstaub, Lärm)

- Endlichkeit der fossilen Brennstoffe

- Zeitverluste durch Staus

- Parkplatzprobleme in den Ballungsgebieten

- Anstieg der privaten Ausgaben für Mobilität [KuRa12]

- Unfallgefahr

Trend 1 ist in keiner seiner diversen Ausprägungsoptionen Voraussetzung für die Trends 2 oder 3. Er entsteht vielmehr als Reaktion auf die genannten Umweltund Nachhaltigkeitsprobleme des MIV. Deswegen sind alle weiteren Ausführungen zu Trends 2 und 3 grundsätzlich unabhängig davon, welche innovativen Antriebstechnologien [DiMA09] sich langfristig durchsetzen werden. In der weiteren Diskussion gehen wir dafür bis auf weiteres von batteriegespeisten Elektrofahrzeugen aus. Die bisherigen Sharing-Konzepte [HePö17], [RüBi15] sind nur als erste Schritte von Trend $3 \mathrm{zu}$ sehen, für dessen Durchbruch offenbar fahrerlose Fahrzeuge die entscheidende Voraussetzung sind. Dieser Zusammenhang wird bisher in der Fachliteratur möglicherweise noch nicht ausreichend gewürdigt.
Diese Kombination erst ermöglicht ein neues Paradigma: Den ,individuellen Massenverkehr".

\section{2.. Grundlagen für den individuellen Massenverkehr der Zukunft}

Die Entwicklungsergebnisse der Trends 1 bis 3 werden es nach allen Prognosen nahezu unvermeidlich ermöglichen, in den kommenden 10-15 Jahren Mobilität in der Form von MaaS sehr umweltschonend, jederzeit (24/7), auf individuelle Anforderung und deutlich preisgünstiger anzubieten als alle bisherigen Angebote, insbesondere als die private PKW-Haltung. Die mittleren Vollkosten für solche Mobilitätsdienste werden heute auf $0,1 €$ je Personenkilometer geschätzt [KuRa12]. Anforderung der Fahrzeuge (baldmöglichst oder zu geplanten Zeiten), Zugangskontrolle und Abrechnung (kilometergenau, per Flatrate oder in gemischten Angeboten) können zuverlässig und personengebunden per App erfolgen.

Solche MaaS-Angebote, die man auch als extreme Form des Car-Sharing oder kleinteiliges Mietwagengeschäft sehen kann, werden, zumindest für die große Mehrzahl derjenigen Menschen, die im Verfügbarkeitsbereich wohnen und arbeiten, wie ein um mehr als eine Größenordnung preiswerterer TaxiDienst funktionieren und dadurch bei etwa gleicher jederzeitiger Verfügbarkeit gegenüber dem Betrieb eines eigenen Kfz viele Vorteile bieten:

- Geringere Kosten (Vollkosten etwa in Höhe des heutigen reinen Benzinverbrauchs)

- Keine Kapitalbindung für Kfz-Anschaffung, kein Wertverlust

- Keine Wartungsverantwortlichkeit, keine Wartungs- und Reparaturkosten 
- Kein Diebstahl-, Vandalismus oder Unfallkostenrisiko

- Keine Parkplatzsuche, -kosten, Wege zum und vom Parkplatz

- Geringeres Verletzungsrisiko durch Unfälle, auch für Dritte

- Keine Fahrzeugführung erforderlich, Fahrzeit anderweitig nutzbar

Die Nachteile sind demgegenüber eher geringfügig: Man kann z.B. persönliche Gegenstände nicht im Fahrzeug lassen, man könnte den Spaß am Selbst-Fahren vermissen, Fahrzeugtyp und Ausstattung (auch Fahrstil) sind nicht mehr geeignet die Persönlichkeit des Fahrers auszudrücken.

Es wird deshalb erwartet, dass leistungsfähige und preiswerte MaaS-Angebote aus wirtschaftlichen Gründen langfristig die Vielzahl der heute als Gebrauchsgegenstand gehaltenen privaten PKW verdrängen wird. Der privat gehaltene PKW wird so von einem Gegenstand des täglichen Gebrauchs zu einer Hobby-bzw. Luxus-Angelegenheit. $\mathrm{Da}$ die MaaSFahrzeuge wesentlich höher ausgelastet werden können als die privat gehaltenen PKW (durchschnittliche Nutzung heute unter einer Stunde je Tag), wird gleichzeitig ein erheblicher Rückgang der Fahrzeuganzahl insgesamt um etwa 80\% erwartet [Flau17]. MaaS-Dienste stützen sich weitgehend auf bereits vorhandene Infrastrukturen ab. Von wesentlicher Bedeutung ist naturgemäß die bereits bestehende Straßeninfrastruktur incl. der Ampelsteuerung. Aber die auch Umstiegspunkte zu anderen Verkehrssystemen können je nach Konzeption eine Rolle spielen. Darüber hinaus ist die Abdeckung mit mobilem Internetzugang, vorzugsweise $5 \mathrm{G}$ und mit GPS bzw. später mit Galileo von Bedeutung.

$\mathrm{Ob}$ sich die für private PKW und Nutzfahrzeuge heute vorhandenen privatwirt- schaftlichen Infrastrukturen für Betankung, Reinigung, Wartung, Reparatur und Sicherheitskontrolle der Fahrzeuge auf die neuen Fahrzeuge und Bedarfe einstellen können, scheint dagegen weniger eindeutig. Das betrifft z.B. 204 Tankstellen im Hamburger Stadtgebiet sowie Kfz-Werkstätten und Einrichtungen zur Überprüfung der Fahrzeugsicherheit, aber auch Pannen- und Abschleppdienste.

Ein konkurrierendes Konzept hierfür wären weniger, aber größere, spezialisierte Betriebshöfe der MaaS-Betreiber. Damit können Rationalisierungsgewinne durch die zu erwartende Standardisierung der Fahrzeuge vermutlich besser ausgeschöpft werden (etwa durch vollautomatisierten Austausch und Aufladung der Batterien) und auch die erforderliche neue Infrastruktur für elektrische oder andere alternative Energieversorgung der Fahrzeuge wirtschaftlicher aufgebaut werden.

Parallel dazu wird eine Daten-infrastruktur erforderlich, die unter anderem Positionen und Status der Fahrzeuge überwacht, die Anforderungen zuteilt, die Nutzung autorisiert und abrechnet und die Leerfahrten minimiert. Hierzu ist neben zentralen Anwendungen und lokaler Intelligenz in den Fahrzeugen eine zuverlässige Kommunikation mit den Fahrzeugen erforderlich.

Natürlich wird es für MaaS grundsätzlich die gleiche Problematik wie bei allen infrastrukturgestützten kurzreichweitigen Diensten geben: Unterschiede zwischen dichter und dünner besiedelten Gebieten hinsichtlich Bedarf, Investitionserfordernissen, Leistungsfähigkeit und Wirtschaftlichkeit des Angebots werden zunächst zu Verfügbarkeitsinseln in oder nahe bei Bereichen mit ohnehin schon starker Infrastruktur führen. Ähnlich wie 
bei Mobilfunknetzen wird die Erschließung von 50\% der Fläche und vielleicht $80 \%$ der Bevölkerung technisch leicht und wirtschaftlich attraktiv sein „der Rest" wird teuer und ggf. in gewissen Zonen wegen Unwirtschaftlichkeit unterbleiben.

\section{Unterschiedliche Szenarien für die Entwicklung von MaaS}

Wie bei allen Innovationen, die von großen Investitionen, insb. mit Infrastrukturaufbau und der Beteiligung breiter Bevölkerungsschichten abhängig sind, ist auch für die Entwicklung von MaaS davon auszugehen, dass das Entwicklungsergebnis weniger durch ein Optimum oder gar Gleichgewicht technologischer oder wirtschaftlicher Qualitäten bestimmt, sondern von einer starken Pfadabhängigkeit (einen guten Überblick gibt z.B. [Pier00]) geprägt werden wird. Wer zuerst in der Lage ist entscheidende Weichenstellungen vorzunehmen, wird die Richtung der Entwicklung wesentlich bestimmen. Deswegen erscheint es sinnvoll, sich bereits heute jedenfalls einige Exponenten der möglichen Entwicklungspfade in Form von Szenarien vor Augen zu führen, die auf gesellschaftliche Kräfte zurückgehen, die $\mathrm{zu}$ solchen Weichenstellungen in der Lage sind. Dazu dürften mit Sicherheit die Autoindustrie, die Internetindustrie und die überwiegend staatlichen oder staatlich regulierten Transportdienstleister des früheren Massenverkehrs gehören. Deswegen werden sich die Entwicklungsergebnisse in dem Raum einstellen können, den die Interessen und Möglichkeiten dieser drei Player aufspannen. Wir skizzieren als Eckpunkte dafür drei Szenarien, in denen jeweils einer der drei Player alle wesentlichen Weichenstellungen allein bestimmt.

\section{1 „MaaS als letztes“}

Autoindustrie und ihre Kunden halten an ihrem langjährig erfolgreichen Grundsatz privater PKW fest. Die Automatisierung wird als Fahrerassistenzsystem so lange als teures Zubehör weiter entwickelt und verkauft, bis endgültig unübersehbar ist, dass ein Fahrer nicht mehr gebraucht wird, sondern nur noch stört. Der Gesetzgeber bleibt bei der Verantwortlichkeit von Halter und Fahrer (am Ende nur noch des Halters) und erteilt die Betriebserlaubnis für den fahrerlosen Betrieb nach technischem Einzelnachweis. Erst am Ende dieser Entwicklung kann es wenigstens zu einem gewissen Rationalisierungspotenzial durch MaaS kommen: Die endlich fahrerlos fahrenden Fahrzeuge können, falls und zu Zeiten, wenn der Eigentümer das gestattet, gegen Entgelt und vermittelt über einen Web-Service auch von anderen Mitnutzern genutzt werden. Die dafür erforderlichen Webservices gibt es heute schon fast, Uber oder Wunder z.B. müssten dazu nur geringfügige Änderungen an ihren bereits bestehenden Lösungen vornehmen. Hinsichtlich der Mobilitätskosten gibt es in diesem Szenario jedoch wesentliche Nachteile. Die Bindung der Fahrzeuge an die Prioritäten der Eigentümer und die Schwierigkeit zu einer Standardisierung und Einfachheit der Fahrzeuge und der erforderlichen Infrastrukturen $\mathrm{zu}$ kommen verhindern hier die Ausschöpfung großer Rationalisierungspotenziale. Auch die Zahl der Fahrzeuge wird nicht sinken. „Stärke“ dieses Szenarios ist, dass niemand in Deutschland wegweisende und damit politisch riskante Entscheidungen zu treffen braucht. 


\section{2 „Maximale McDonaldisierung [Ritz13] des MIV durch globale Internetfirmen“"}

Die heutigen Betreiber von Drive-Sharing Services oder andere potente XaaSAnbieter entwickeln ihre ITK-Lösungen in Richtung MaaS weiter und lassen sich standardisierte fahrerlose Fahrzeuge und spezielle Infrastrukturkomponenten nach eigener Spezifikation von „Hoflieferanten" entwickeln (etwas weniger komplexe Parallele ist der heutige Smartphone-Markt). Erwerb, Wartung, Reinigung und Betrieb der Fahrzeuge wird potenten lokalen staatlichen oder privaten Unternehmen als Lizenz oder Franchise-Vertrag verkauft, verbunden mit einem Flächendeckungsziel für den Infrastrukturaufbau. Vorteil ist hier die volle Ausschöpfung aller Rationalisierungspotenziale bei globalem Multiplikationspotenzial - allerdings nicht unbedingt ihre Weitergabe an die Kunden. Der „Legacy-ÖPNV“ als von der Transportleistung her vergleichsweise kleiner, subventionierter Wettbewerber dürfte hierbei unter enormen Druck geraten. Know-How, sämtliche Kundendaten, der überwiegende Teil der Wertschöpfung und die Technologieführerschaft verbleiben beim Franchisegeber. Auch hier ist eine "Stärke“ des Szenarios, dass in Deutschland keine wegweisenden und damit politisch riskanten Entscheidungen erforderlich sind.

\section{3 ,ÖPNV 4.0“}

Die heutigen Betreiber von ÖPNV-Angeboten versuchen wieder Massenverkehrsanbieter $\mathrm{zu}$ werden. Dazu decken sie einen möglichst großen Anteil des Potenzials des bisherigen MIV durch die Entwicklung eigener MaaS-Lösungen $\mathrm{ab}$, die sie nahtlos in ihr bisheriges
ÖPNV-Angebot Angebot integrieren können. Auch sie lassen sich standardisierte fahrerlose Fahrzeuge, IT- und Infrastrukturkomponenten passend entwickeln, betreiben aber den gesamten Dienst in eigener wirtschaftlicher und technischer Verantwortung. Neben der unternehmerischen Autonomie wäre ein wesentlicher Vorteil, dass hier gesetzliche Regelungen zur Verarbeitung personenbezogener Daten gegenüber den $\mathrm{Be}-$ treibern durchgesetzt werden könnten. Um möglichst hohe Stückzahlen für attraktive Angebote der Fahrzeugindustrie und der IT-Industrie zu erreichen, müssten die Verkehrsbetreiber allerdings über ihre nationalen und internationalen Verbände Abstimmung und Standardisierung betreiben und Einkaufsgenossenschaften bilden. Vorteilhaft wäre weiter, dass ÖPNV allgemein als Bestandteil der Daseinsvorsorge gilt und daher als staatlicher Obhut, insbesondere Regulierung und Subventionierung zugänglich angesehen wird.

\section{Fazit}

Die drei genannten Varianten sind insofern extrem, als in jeder jeweils einer der großen Player allein den Kurs bestimmt. Natürlich sind auch andere Entwicklungspfade möglich, insbesondere national unterschiedliche sowie Zwischenstufen zwischen den genannten Extremen oder Varianten durch den Einfluss anderer Player. Wie bei allen pfadabhängigen Entwicklungen ist aber zu erwarten, dass es die Aktivitäten des ersten bedeutenden Players sein werden, die wesentliche Weichen für die Entwicklung der neuen Technologie stellen. So unvollkommen dessen Lösung auch zunächst sein mag, wird man hinter diese später auch mit ggf. besseren Lösungen nur schwer zurückkommen. In einer sol- 


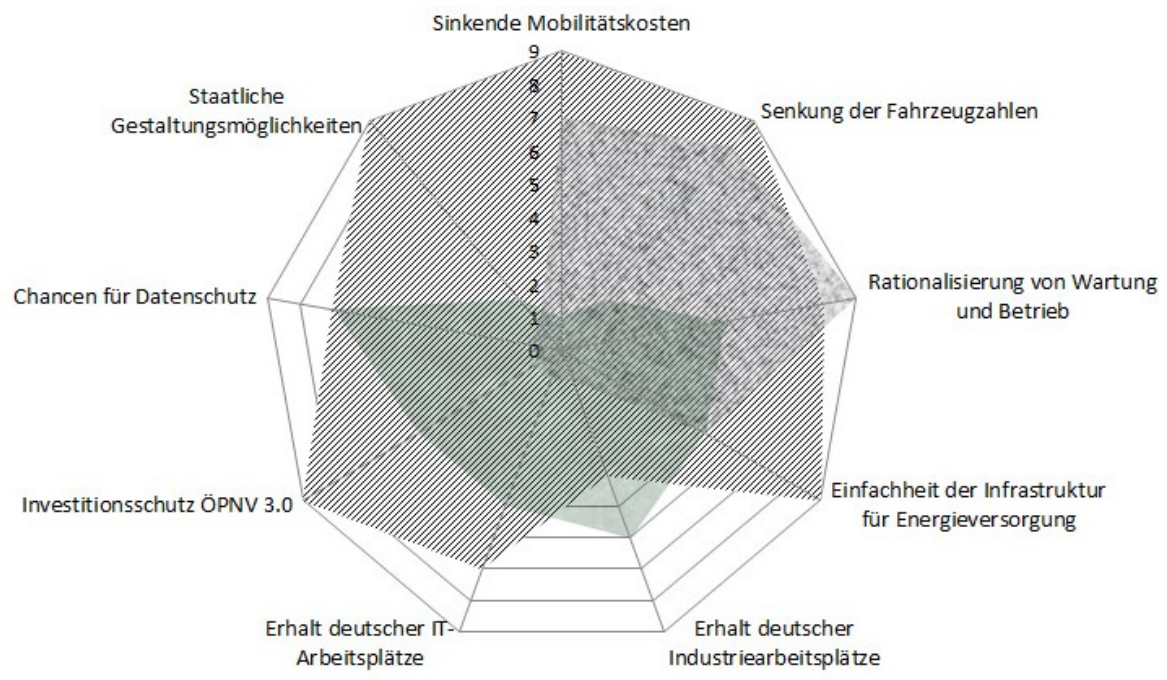

॥̈ ÖPNV 4.0 maximale McDonaldisierung Mast

Abbildung 1: Abb. 1: Qualitativer Vergleich der drei Szenarien nach der Ausprägung von 9 Parametern, $0:=$ gar nicht, 9:= maximal

chen Situation kann es durchaus sein, dass wertvolle Entscheidungsspielräume durch Abwarten verloren gehen und der gewinnt, der sich zuerst energisch bewegt.

Deswegen engagieren sich die Autoren in der Initiative Hamburger Auto-Transit für Lösungen vom Typ ÖPNV4.0, in der sich der ÖPNV den überwiegenden Teil der Verkehrsleistung des MIV in Form des individuellen Massenverkehrs zurückerobert. Dabei handelt es sich jedoch auch für eine einzelne, kleinere Metropolregion wie Hamburg bereits um eine Entwicklung mit einem Investitionsvolumen in zehnstelliger Höhe, die mutige politische Entscheidungen erfordert. Global gesehen handelt es sich um eine der größten technologischen Revolutionen, die durchaus keinen Vergleich mit der Smartphone-Revolution zu scheuen braucht und größte Aufmerksamkeit verdient.

\section{Literaturverzeichnis}

[Hütt13]

Hütter, A.: Verkehr auf einen Blick. Statistisches Bundesamt, Wiesbaden, 2013

[Beck11]

Becker, U.: Verkehr und Umwelt. In: Schwedes, O. (Hrsg.): Verkehrspolitik, VS Verlag, Wiesbaden, 2011, S. 77-89.

[BrPe17]

Brivio, E., Petsa, I.: Kommission droht Deutschland, Frankreich, Spanien, Italien und dem Vereinigten Königreich mit Klage wegen anhaltender übermäßiger Luftverschmutzung, 2017, http://europa.eu/rapid/press-release_IP-17-

238_de.htm (Abruf 18. Feb. 2017)

[KuRa12]

Kunert, U., Radke, S.: Personenverkehr in Deutschland: Mobil bei hohen Kosten, DIW-Wochenbericht, ISSN 0012-1304, Vol. 79, Iss. 24, 2012, S. 3-12 
[DiMA09]

Dierkes, M., Marz, L., Aigle, T.: Die automobile Wende. In: Popp, R, Schüll, E. (Hrsg.): Zukunftsforschung und Zukunftsgestaltung, Springer, Berlin, 2009, S. 323-340

[HePö17]

Heinen, J. H., Pöppelbuß, J.: Elektromobilität im gewerblichen Car-Sharing. In: Thomas, O., Nüttgens, M., Fellmann, M. (Hrsg.): Smart Service Engineering, Springer Fachmedien, Wiesbaden, 2017, S. 282-302.

[RüBi15]

Rühl, C., Biethahn, N.: Strategische Herausforderungen im Rahmen der Markteintrittsentscheidung von stationsungebundenen Business-to-Consumer Carsharing Anbietern-eine Meta-Analyse. In: Sucky, E., Werner, J., Kolke, R., Biethahn, N. (Hrsg.): Mobility in a Globalised World 2014, University of Bamberg Press, 2015, S. 218-239.

[Flau17]

Flausch, A.: UITP Policy Brief „Autonomous vehicles: a potential game changer for urban mobility”, UITP, Brüssel, 2017, S.2

\section{[Pier00]}

Pierson, P.: Increasing returns, path dependence, and the study of politics. In: American Political Science Review, Vol. 94, 2/2000, S. 251-267

[Ritz13]

Ritzer, G.: The McDonaldization of society, Sage Publications, Thousand Oaks, CA, 2013

\footnotetext{
(C) $10 \begin{aligned} & \text { Dieser Beitrag ist unter de } \\ & \text { Creative-Commons-Lizenz }\end{aligned}$ BY NC ND CC BY-NC-ND lizensiert.
} 